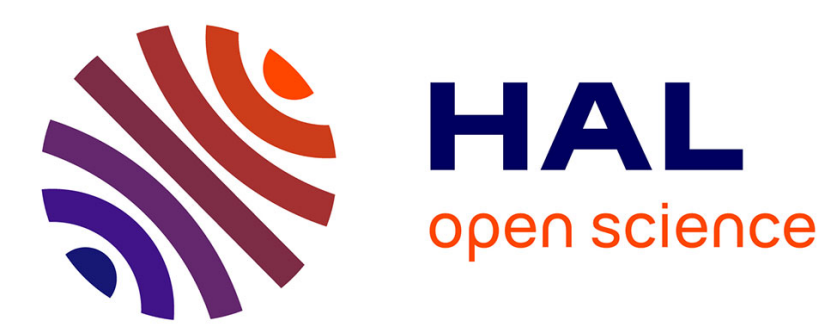

\title{
Irreversible effects in the paramagnetic-state of DyVO4 in the crystallographic distorted phase
}

\author{
B. Daudin, G. Chouteau, N. M'Sirdi
}

\section{To cite this version:}

B. Daudin, G. Chouteau, N. M'Sirdi. Irreversible effects in the paramagnetic-state of DyVO4 in the crystallographic distorted phase. Journal de Physique, 1984, 45 (1), pp.169-175. 10.1051/jphys:01984004501016900 . jpa-00209734

HAL Id: jpa-00209734

https://hal.science/jpa-00209734

Submitted on 1 Jan 1984

HAL is a multi-disciplinary open access archive for the deposit and dissemination of scientific research documents, whether they are published or not. The documents may come from teaching and research institutions in France or abroad, or from public or private research centers.
L'archive ouverte pluridisciplinaire HAL, est destinée au dépôt et à la diffusion de documents scientifiques de niveau recherche, publiés ou non, émanant des établissements d'enseignement et de recherche français ou étrangers, des laboratoires publics ou privés. 
Classification

Physics Abstracts

$64.70 \mathrm{~K}-75.90$

\title{
Irreversible effects in the paramagnetic-state of $\mathrm{DyVO}_{4}$ in the crystallographic distorted phase
}

\author{
B. Daudin \\ SBT/LACC, CEN-G, BP 85 X, 38041 Grenoble Cedex, France \\ G. Chouteau and N. M'Sirdi \\ SNCI and CRTBT, CNRS, BP 166 X, 38042 Grenoble Cedex, France
}

(Reçu le 30 juin 1983, révisé le 5 septembre, accepté le 16 septembre 1983)

\begin{abstract}
Résumé. - Nous avons mesuré la susceptibilité magnétique statique et alternative, l'aimantation et l'atténuation hypersonore de $\mathrm{DyVO}_{4}$ dans la phase paramagnétique et à une température inférieure à celle de la transition Jahn-Teller coopérative. Nous avons observé des phénomènes irréversibles qui dépendent du cyclage thermique de l'échantillon. Nous avons interprété ces effets comme une manifestation de la transformation de l'échantillon d'un état cristallographique polydomaine à un état monodomaine. La grande mobilité des parois de domaine joue un rôle important dans cette transformation.
\end{abstract}

\begin{abstract}
We have studied the low-field magnetic susceptibility (a.c. and d.c.), the magnetization and the hypersonic attenuation of $\mathrm{DyVO}_{4}$ in the paramagnetic state below the cooperative Jahn-Teller transition temperature. Irreversible phenomena depending on the sample thermal cycling are observed. We interpret these effects as due to the transformation from a polydomain to a monodomain crystallographic state favoured by a great mobility of the domain-walls.
\end{abstract}

\section{Introduction.}

$\mathrm{DyVO}_{4}$ belongs to the well-known $\mathrm{RXO}_{4}$ family where $\mathrm{R}$ is a rare earth and where $\mathrm{X}$ is $\mathrm{V}$, As or $\mathrm{P}$. Some of these compounds undergo at low temperature a crystallographic phase transition induced by a cooperative Jahn-Teller effect and were extensively studied for this reason (see for instance the review paper by Gehring and Gehring [1]).

At room temperature, $\mathrm{DyVO}_{4}$ has the tetragonal zircon structure with space group $\mathrm{D}_{4 \mathrm{~h}}^{19}$. At $T_{\mathrm{D}}=14 \mathrm{~K}$, due to the cooperative Jahn-Teller effect, this symmetry is lowered to orthorhombic with space group $\mathrm{D}_{2 \mathrm{~h}}^{28}$ [2] In addition, $\mathrm{DyVO}_{4}$ becomes antiferromagnetic below $T_{\mathrm{N}}=3.05 \mathrm{~K}[2]$.

The lowest energy levels of $\mathrm{Dy}^{3+}$ ion are two Kramers doublets which are separated by an energy $\Delta_{0}=13 \mathrm{~K}$ in the high symmetry phase [3]. Below $T_{\mathrm{D}}$, this separation is increased due to the Jahn-Teller effect and is $39.6 \mathrm{~K}$ at zero kelvin.

Because of the two equivalent crystallographic distortions, the sample has a polydomain structure below $T_{\mathrm{D}}$. As a consequence, it is not possible to define an unique set of $a$ and $b$ axes in the low temperature phase. Nevertheless, it is possible to intervert these two axes by application of a magnetic field or to convert the sample into a monodomain as was experimentally observed [4-5].

The $g$ factor is very anisotropic in the $a-b$ plane. For the ground state doublet, it was found that $g_{1 \mathrm{a}} \simeq 19$ and $g_{1 \mathrm{~b}} \simeq 0$ while for the upper doublet $g_{\mathrm{ua}} \simeq 0$ and $g_{\mathrm{ub}} \simeq 19[4,6]$.

It was also found that the distortion direction is correlated with the direction of principal $g$ value and the effect of a magnetic field is to make an unique $a$-axis prevail for the whole crystal [4].

Magnetically, the system is in a paramagnetic-state above and below $T_{\mathrm{D}}$ but magnetic susceptibility anomalies are observed due to the change in the energy levels separation which is associated to the crystallographic transition [7-10].

Measurements by previous authors revealed two puzzling points :

- a magnetization hysteresis was observed and measured as a function of magnetic field for $T_{\mathrm{N}}<T<T_{\mathrm{D}}$. The interpretation of this was not satisfactory $[8]$; 
- discrepancies between the various magnetic susceptibility results occur and are apparently due to the change in measurement frequency value (d.c. or a.c. measurements) [7-10].

The aim of this paper is to clarify this last point and determine the process responsible for the observed hysteresis. All the experiments were performed for $T>4.2 \mathrm{~K}$, that is in the paramagnetic phase.

\section{Experimental results.}

1.1 Technical arrangement. - The samples used for the experiments were provided by S. H. Smith, from the Clarendon Laboratory in Oxford. They were prepared by the flux method and their typical dimensions are of $5 \times 1 \times 1 \mathrm{~mm}^{3}$. The dislocations densities were found to be low by X-rays measurements. Nevertheless, flux inclusions may be present in the crystal and far higher dislocation densities were found around them $[11,12]$.

Static magnetization measurements were performed using a vibrating pick-up coil technique. The apparatus was recently automatized and the sensitivity was then greatly increased [13]. The a.c. susceptibility was also measured on the same sample at various frequencies using a classical mutual inductance bridge device. For all the experiments the specimen was mounted strain free in the sample-holder and no damages due to thermal cyclings were observed.

1.2 RESULTS IN ZERO FREQUENCY. - The magnetization was first measured as a function of magnetic field for various temperatures below and above $T_{\mathrm{D}}$. The experimental procedure was to first cool all the device down to $4.2 \mathrm{~K}$ in zero magnetic field. Then the sample temperature was regulated at the chosen value and once the equilibrium was reached, the magnetization was measured from $H=0$, to $H$ final.

We have performed a few measurements up to $120 \mathrm{kOe}$ at various temperatures. At $4.2 \mathrm{~K}$ the magnetization $M(H)$ saturates at $H \simeq 20 \mathrm{kOe}$. In high-fields the variation is linear and given by :

$$
M(H)=M_{0}+a H
$$

where $M_{0} \simeq 5 \times 10^{4} \mathrm{emu} / \mathrm{mole}$ and $a \simeq 50 \mathrm{emu} /$ mole.kOe. The $a H$ term is presumably due to the higher energy levels of the multiplet.

From the $M_{0}$ value, it is possible to calculate the magnetic moment of the ground state of the $\mathrm{Dy}^{3+}$ ion. The result is $\mu=8.96 \mu_{\mathrm{B}}$, were $\mu_{\mathrm{B}}$ is the Bohr magneton. This value is in agreement with previous results deduced from Mössbauer [14], neutron diffraction [15] and magnetization [8] measurements.

The detailed behaviour in low magnetic field is shown in figure 1 for $T=4.2 \mathrm{~K} ; 5.6 \mathrm{~K}$ and $16.5 \mathrm{~K}$ respectively. The magnetic field has been corrected from the demagnetizing effects.

The most prominent feature is the presence of a large hysteresis at $T_{\mathrm{N}}<T<T_{\mathrm{D}}$ whereas this effect is not present for $T>T_{\mathrm{D}}$.

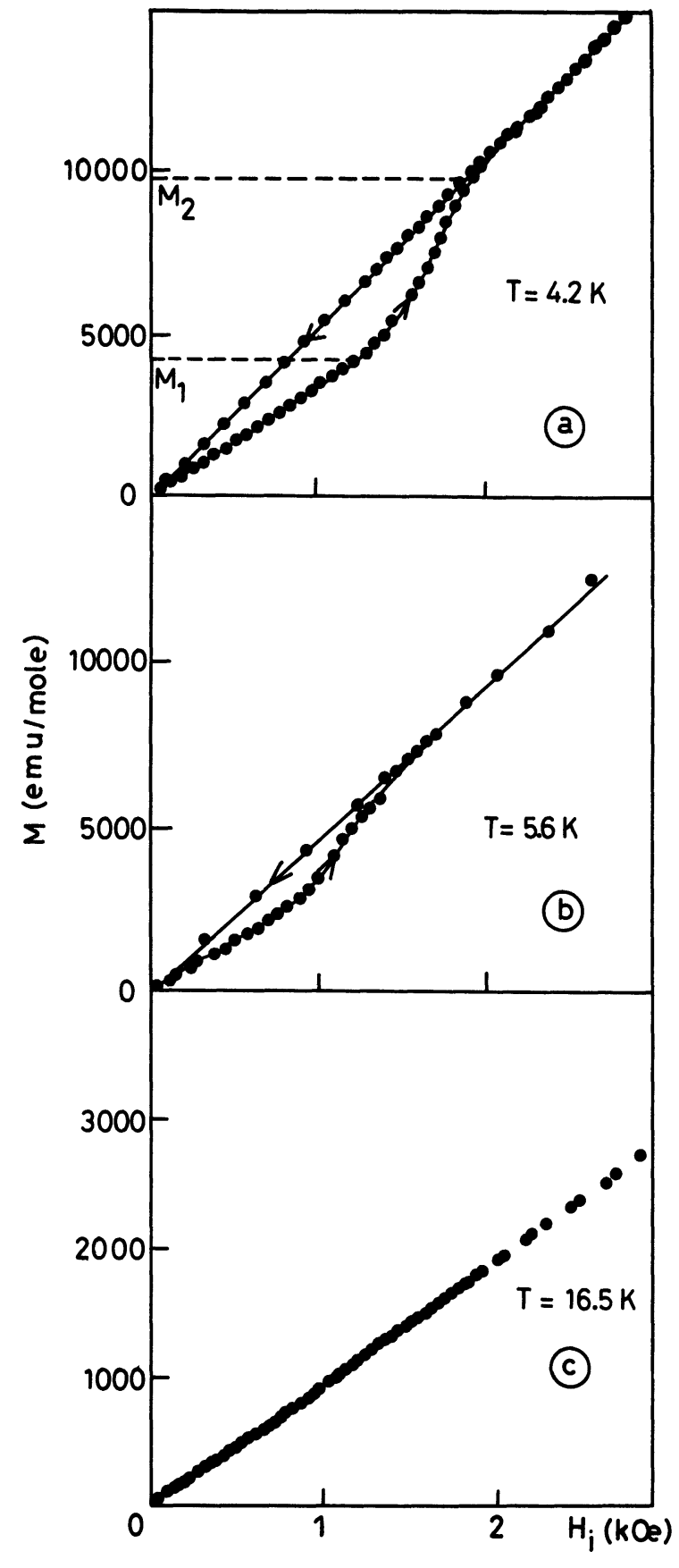

Fig. 1. - Magnetization versus internal magnetic field showing the disappearance of hysteresis loop above $T_{\mathrm{D}}$. a) $T=4.2 \mathrm{~K}$; b) $T=5.6 \mathrm{~K}$; c) $T=16.5 \mathrm{~K}$.

In figure $1 a$ we define the two magnetization values from each side on the non linear region : we find that $M_{1}=4200 \mathrm{emu} / \mathrm{mole}$ at $H_{1}=1246 \mathrm{Oe}$ and $M_{2}=9800 \mathrm{emu} / \mathrm{mole}$ at $H_{2}=1970 \mathrm{Oe}$.

We then performed another measurement changing the experimental procedure $: a$ ) first, the sample was warmed up to $20 \mathrm{~K} ; b$ ) a $20 \mathrm{kOe}$ magnetic field was then established and the sample was cooled down to $4.2 \mathrm{~K}$ in presence of this magnetic field ; $c$ ) the magnetic field was reduced to zero and afterwards the magnetization was measured from $H=0$ to $H$ final. 
No hysteresis was observed in this second experiment.

Unambiguously, this indicates that the hysteresis is due to the coexistence of two crystallographic domains types up to a magnetic field large enough to switch the "misoriented" domains and convert the sample into a monodomain. Such a reorientation of the domains has been already observed by previous authors $[4,5,21]$.

In the second experiment, the sample is cooled in the presence of a magnetic field which breaks down the equivalence between the two possible distortions so that the sample is monodomain below $T_{\mathrm{D}}$. When the magnetic field is then reduced to zero, this monodomain state is preserved and no magnetization hysteresis is observed.

If the two domain classes were completely equivalent, one would expect that $M_{2} / H_{2} \simeq 2 M_{1} / H_{1}$. This is not actually observed, which indicates that some distortion is favoured during the transition.

The magnetization was then measured as a function of the temperature. If the magnetic field is small enough, it is possible to deduce in this way the magnetic susceptibility assuming that

$$
\frac{M(T)}{H} \simeq \chi \simeq \frac{\partial M(T)}{\partial H} .
$$

The result is shown in figure 2 for two different magnetic fields. For $H=862 \mathrm{Oe}$, two anomalies are observed :

a) A complicated behaviour around $T=14 \mathrm{~K}$ which is associated to the crystallographic phase transition.

b) A second anomaly at $T=8 \mathrm{~K}$ which is associated with an hysteresis effect and is attributed to the conversion of the sample into a monodomain.

The second magnetization curve in figure 2 was measured for $H=358 \mathrm{Oe}$ and for a monodomain sample : the anomaly around $14 \mathrm{~K}$ is still present but the second anomaly at $T \simeq 8 \mathrm{~K}$ vanished as was expected.

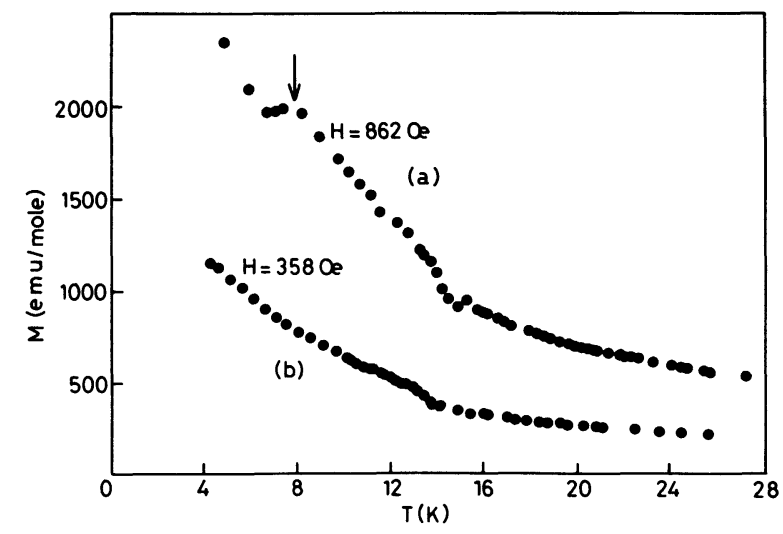

Fig. 2. - Magnetization versus temperature. a) $H=862 \mathrm{Oe}$, polydomain state. b) $H=358 \mathrm{Oe}$, monodomain state.
1.3 A.C. MEASUREMENTS RESUlts. - In order to understand the differences between previously published susceptibility results that we mentioned in the introduction of this paper, the real and imaginary parts of the magnetic susceptibility were then measured at various frequencies.

In a preliminary experiment, the frequency dependence of $\chi^{\prime}$ and $\chi^{\prime \prime}$ was measured for $T=4.2 \mathrm{~K} \ll T_{\mathrm{D}}$ and $T=11 \mathrm{~K}$ close to $T_{\mathrm{D}}$.

In the range $11 \mathrm{~Hz}-20 \mathrm{kHz}$ no effect was found either for $\chi^{\prime}$ or for $\chi^{\prime \prime}$ similarly to previous results [22]. In both experiments $\chi^{\prime \prime}$ was found to be several orders of magnitude smaller than $\chi^{\prime}$ so that actually $\chi \simeq \chi^{\prime}$. These results are an indication that the crystallographic domain relaxation, if it exists, does not occur in this frequency range.

In further experiments, the susceptibility was measured as a function of the temperature. Typical results are shown in figure 3 . Note that, for all these experiments, $\chi$ is expressed in arbitrary units and not in emu.

Run (1) : the first measurement was performed at $v=100 \mathrm{~Hz}$ and the result is qualitatively very similar to the previous results obtained at $v=0$ (see Fig. 2).

Run (2) : the sample was then cooled down from $18 \mathrm{~K}$ to $4.2 \mathrm{~K}$ in zero-field and a measurement was performed at $v=500 \mathrm{~Hz}$. It appears that the qualitative behaviour of $\chi^{\prime}$ is similar to that observed for run (1) but the absolute value of $\chi^{\prime}$ is smaller for $T<T_{\mathrm{D}}$. An important point is that the curves join themselves above $T_{\mathrm{D}}$.

Run (3) : the sample was then cooled down again from $18 \mathrm{~K}$ to $4.2 \mathrm{~K}$ and $\chi^{\prime}$ was measured for $v=1000 \mathrm{~Hz}$. It was found that the qualitative behaviour of $\chi^{\prime}$ was considerably modified in this experiment as shown in figure 3 : in particular a plateau which extends roughly from $12.5 \mathrm{~K}$ to $14.5 \mathrm{~K}$ was observed.

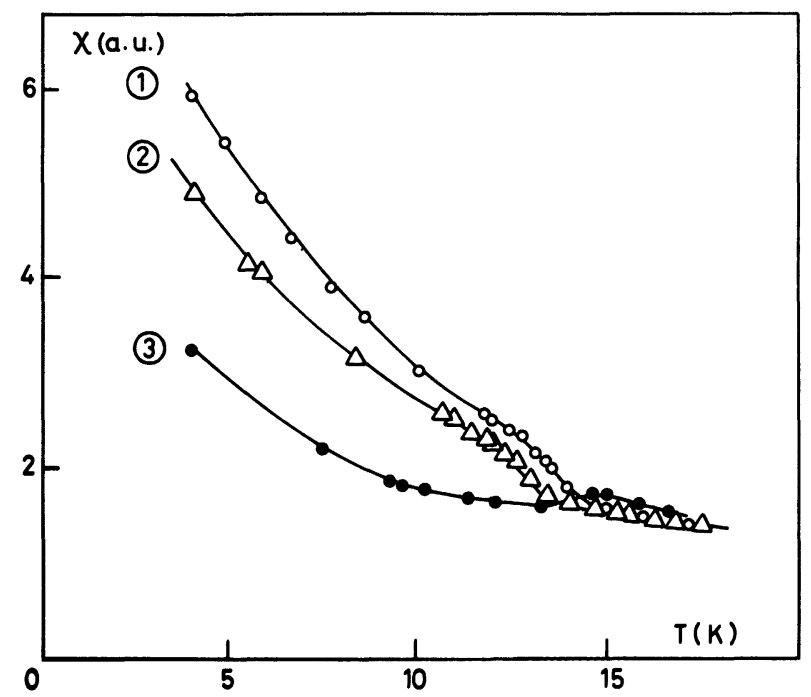

Fig. 3. - a.c. susceptibility versus temperature. 1, 2, 3 : see text. 
Run (4) : the sample was cooled down from $18 \mathrm{~K}$ to $4.2 \mathrm{~K}$ and $\chi^{\prime}$ was measured again for $v=100 \mathrm{~Hz}$. The high $\chi^{\prime}$ value obtained during the first experiment is not recovered. So far as the general shape of $\chi^{\prime}$ is concerned, it is very similar to that observed for $v=1000 \mathrm{~Hz}$.

These experiments show clearly that various states are possible for the sample : the evolution from one state to another appears to be frequency insensitive but it is governed by thermal cycling through $T_{\mathrm{D}}$, suggesting that defects play a rôle.

Hence, in a further step of the experiments, the sample was annealed at $77 \mathrm{~K}$ during $12 \mathrm{~h}$ and measured again. The result is shown in figure 4 for $v=100 \mathrm{~Hz}$ and $v=11 \mathrm{~Hz}$. Although the behaviour close to the transition is complex, a point of interest is that the absolute value of $\chi^{\prime}$ at $4.2 \mathrm{~K}$ still remains low and the value obtained during the first run (see Fig. 3) is not recovered.

On the other hand it is worthwhile noting that the anomaly associated to the transition is present at temperatures as low as $11 \mathrm{~K}$, compared to $12.5 \mathrm{~K}$ for the first experiment.

To sum up at this part of the work, two major conclusions may be deduced from the experiments which have been described :

i) The change in the $\chi^{\prime}$ behaviour is not a frequency effect but is associated to the cycling of the sample above and below $T_{\mathrm{C}}$.

ii) A stabilization to a " final " state is observed and the anomaly in $\chi^{\prime}$ is shifted to the lowest temperatures.

The sample was then annealed at high temperature in the usual way [16] : it was kept at $1200^{\circ} \mathrm{C}$ during one hour and slowly cooled down to room temperature at a rate of about $1.5 \mathrm{~K} / \mathrm{min}$.

$\chi^{\prime}$ was then measured for $v=100 \mathrm{~Hz}$ and the result is shown in figure 5 . The high $\chi^{\prime}$ value at $4.2 \mathrm{~K}$ is not yet recovered and the general behaviour is similar to that previously observed but a significant feature is that the $\chi^{\prime}$ anomaly is observed above $12 \mathrm{~K}$, that is $1 \mathrm{~K}$

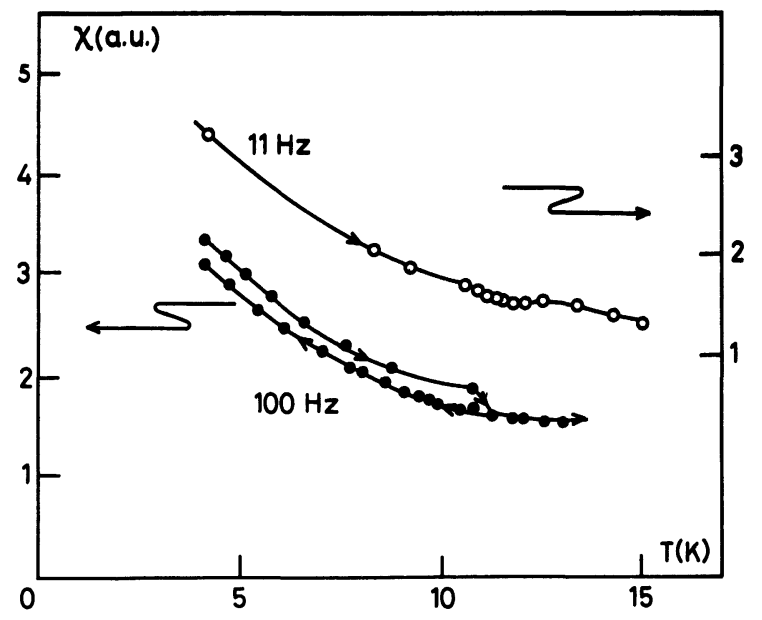

Fig. 4. - a.c. susceptibility versus temperature after annealing at $77 \mathrm{~K}$. The vertical axis has been shifted for clarity.

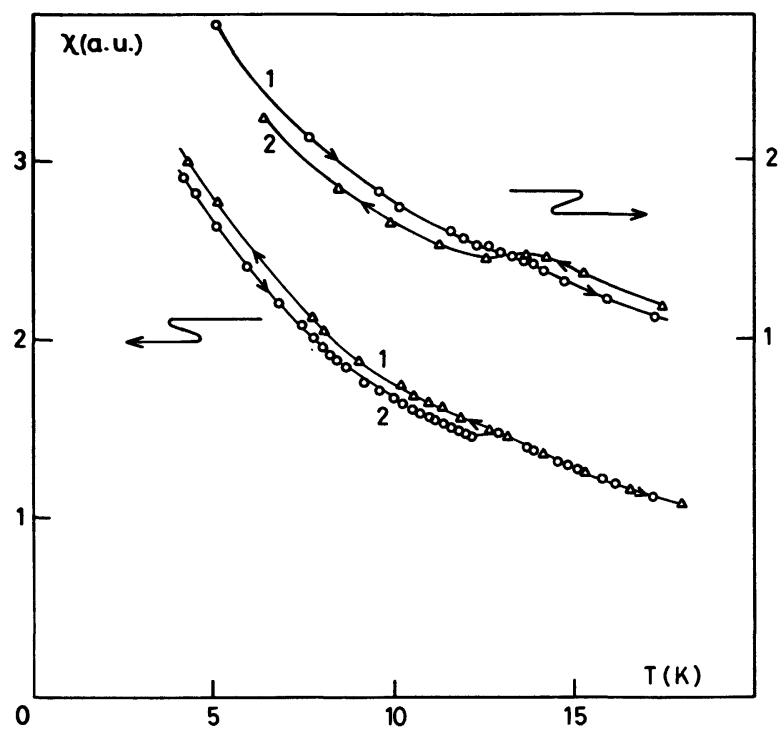

Fig. 5. - a.c. susceptibility versus temperature after annealing at $1200^{\circ} \mathrm{C}$. The vertical axis has been shifted for clarity.

higher than in the previous experiment reported in figure 4.

This result is puzzling. It shows that the domains walls always appear in the same general configuration below $T_{\mathrm{D}}$ provided a sufficient number of thermal cycling through $T_{\mathrm{D}}$ has been made.

A new experiment was therefore carried out after a $\pi / 2$ rotation of the sample with respect to its orientation during all the experiments previously described. It is worth stressing that for a perfect crystal this new orientation is completely equivalent to the first one. In the case of our experiments, the sample had a rectangular rather than a square section and the short side of the rectangle was now parallel to the magnetic field direction. The susceptibility as a function of $T$ and also the magnetization as a function of $H$ were therefore measured in this new geometry. The results are shown in figures 6-I and 6-II.

So far as magnetization is concerned (Fig. 6-II), the behaviour is basically similar to previous measurements but it is worth making three remarks :

i) Below $1.7 \mathrm{kOe}$, the magnetization is quite low and the conversion to the monodomain configuration for $H=1.7 \mathrm{kOe}$ is very rapid as indicated by the sharpness $(\Delta H \simeq 100 \mathrm{Oe})$ of the magnetization step around this $H$ value.

ii) The transformation is yet not completed above $H=1.8 \mathrm{kOe}$ and a linear variation for $M$ is not observed until a rather high magnetic field is reached. In addition, and as a confirmation of this effect, another step is observed on $M$ at $H=2.6 \mathrm{kOe}$ when the magnetic field is lowered down to zero. Once this second transformation was completed, it was checked that the magnetization variation was the same as that shown in figure 1 for the monodomain configuration. 


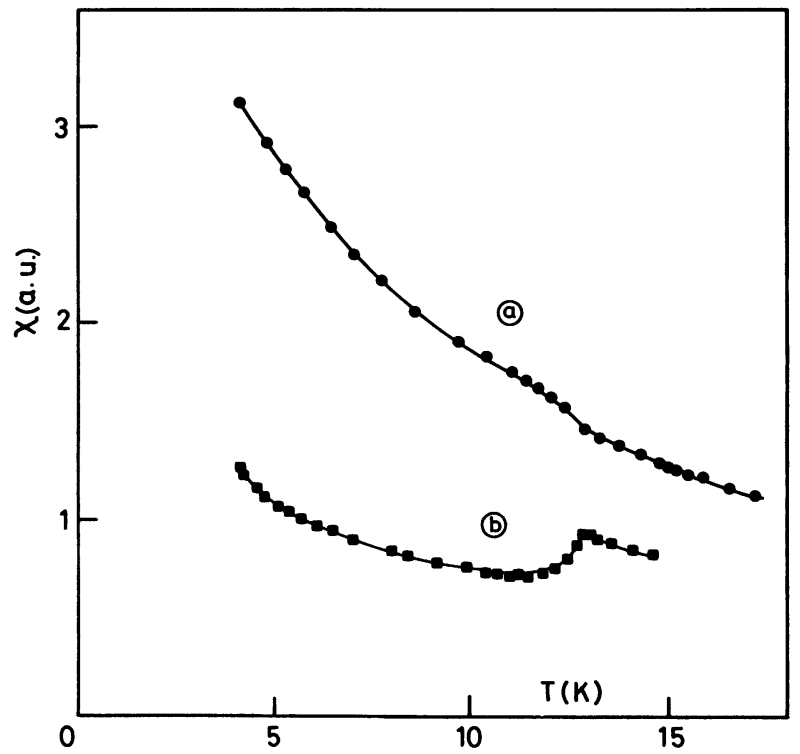

(I)

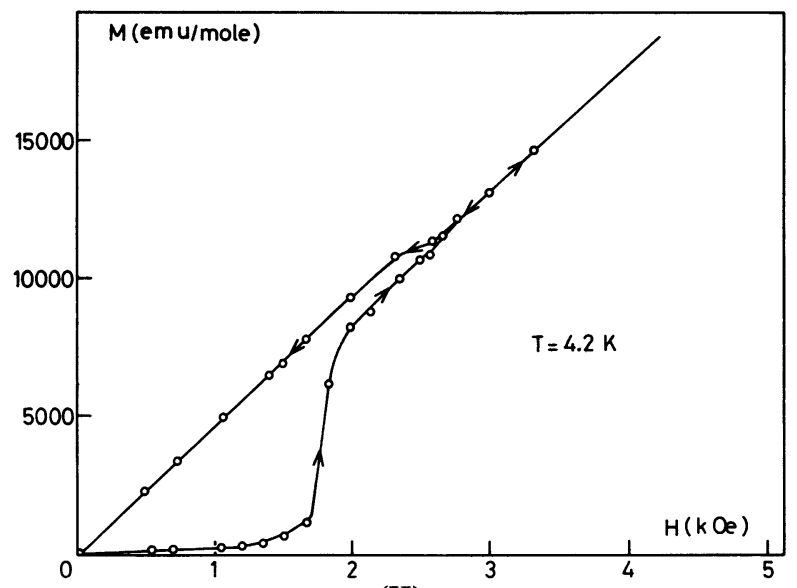

(II)

Fig. 6. - a.c. susceptibility versus temperature (I) and magnetization versus field (II) after a $\pi / 2$ sample rotation. Curve $a$ in figure 6-I was obtained before a $\pi / 2$ sample rotation and is shown for comparison.

iii) After several thermal cyclings through $T_{\mathrm{D}}$, the initial magnetization slope increases and the magnetization step becomes subsequently smaller and smaller. Finally it completely disappears and the magnetization curve corresponds to the monodomain situation which is stabilized this way.

The results for magnetic susceptibility measured along the new direction are also shown in figure 6-I, curve $b$. It is clear that the variation close to $T_{\mathrm{D}}$ is very different from the experiments which were performed in the previous geometry. Nevertheless, the result is consistent with magnetization measurements and indicates surprisingly that the domain orientation characterized by a principal $g$ value direction perpendicular to the magnetic field is favoured in this case.

This is particularly spectacular for the magnetization and the large step indicates that a major part of the sample with $g \simeq 0,5$ is suddenly converted to a $g \simeq 19$ region in contrast with the previous experiments where the conversion was smoother. This indicates that the initial domain distribution through the sample was roughly equally balanced between the two possibilities.

Our interpretation of these two experiments is that nucleation conditions are clearly demonstrated to be determinant in the properties of $\mathrm{DyVO}_{4}$ and surface effects or pre-strains present in the high temperature phase will drastically influence the structure of the low temperature phase.

\section{Interpretation and discussion.}

The original theoretical description of $\mathrm{DyVO}_{4}$ was done by Elliott et al. [3]. The molecular field theory developed by these authors was able to explain qualitatively their experimental results. Nevertheless it failed to predict the correct transition temperature and to describe the experimental results in the close vicinity of $T_{\mathrm{D}}$. This is due to the fact that the short range order interactions can not be correctly taken into account in a molecular field approximation.

In an attempt to improve the theoretical description, it was recently shown that a " compressible Ising model " type correction could be successfully made in zero magnetic field. Such a modified theory was found to account well for the first order transition in $\mathrm{DyAsO}_{4}$ [17].

It was furthermore found that the specific heat measurements of $\mathrm{DyVO}_{4}$ in zero magnetic field also could be described satisfactorily in this way [18]. Nevertheless, the model failed to explain the specific heat measurements performed in presence of magnetic field.

On the other hand Kasten using a simple molecular field theory was able to give a qualitatively correct description of the susceptibility in the monodomain case. The "compressible " Ising model was found to give a similar result [19].

In the real case, the measured $\chi$ is given by

$$
\chi=V_{1} \chi_{a}+V_{2} \chi_{b}
$$

where $V_{1}$ and $V_{2}$ are the respective number of domains with the principal $g$-value along $a$ or $b$. Therefore, one has to find the equilibrium conditions of the domains, which is a very difficult task [20].

An additionnal important parameter of the problem is the domain walls mobility which governs the transformation from the polydomain to the monodomain state. Furthermore the mobility is deeply influenced by the pinning conditions leading to the irreversible phenomena that we observed.

An evidence of this is provided by hypersonic attenuation experiments which were performed in addition to the magnetic experiments.

Hypersonic measurements : The details of the experimental arrangement are given elsewhere [19]. The sample was larger than the one used for magnetic measurements. It was a parallelepiped of $1.3 \times 1.9 \times$ 
$4.8 \mathrm{~mm}^{3}$ oriented along the $c$-axis. Its ends were carefully polished. It was glued to a piezoelectric quartz transducer which was placed in the maximum electrical field zone of a reentrant cavity. The phonons produced were reflected at the end of the $\mathrm{DyVO}_{4}$ sample and the intensity of the resulting "echoes " were measured as a function of the magnetic field.

The propagation of the hypersonic wave was along the $c$-axis and the magnetic field could be applied along any arbitrary direction in the $a-b$ plane, perpendicular to the $c$-axis.

A typical spectrum is shown in figure 7 for our arbitrary direction of the magnetic field with respect to the $a$-axis. The three prominent features are :

i) an important hysteresis;

ii) a very large attenuation above $15 \mathrm{kOe}$;

iii) the presence in the high field region of the hysteresis cycle of a noise much more important than the usual experimental noise which is visualized in the low field region of the cycle.

In the lower part of figure 7 the result for an incomplete cycle is shown : the extra noise is clearly present and vanishes when the field is reduced to about $9 \mathrm{kOe}$.

The effect of the orientation of $H$ with respect to the crystallographic axes was analysed and the field $H_{\mathrm{p}}$ associated with the high field region of the hysteresis cycle was plotted as a function of $\theta$.

It is worthwhile noting that the position $\theta=0$ was arbitrary and that, due to the experimental procedure,

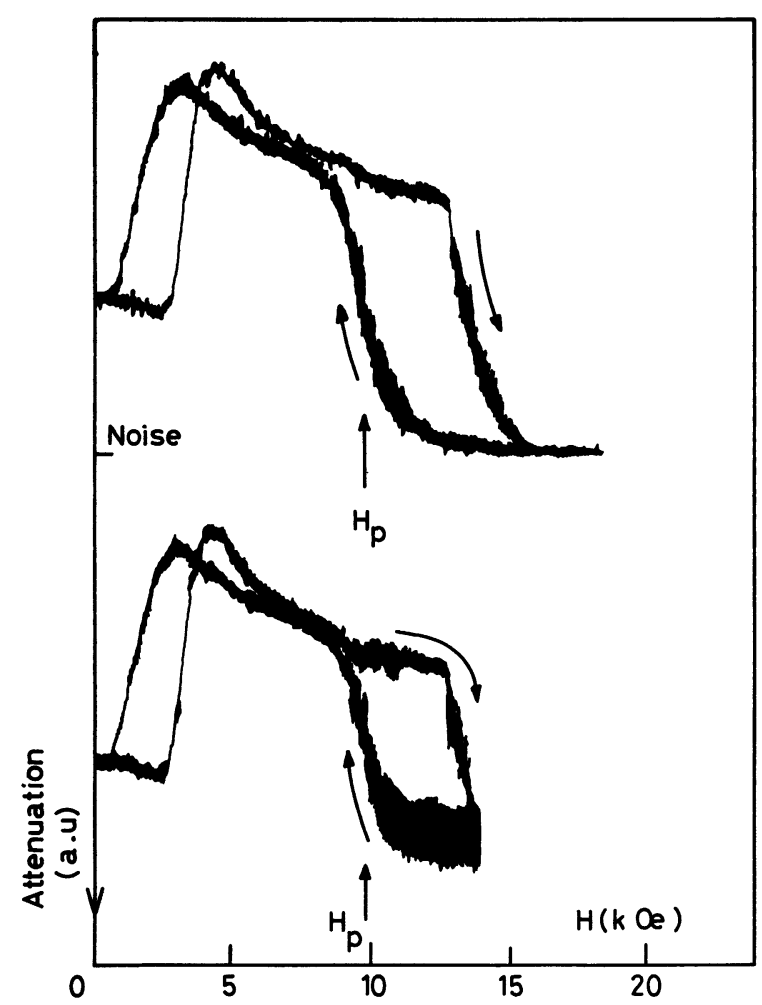

Fig. 7. - Hypersonic attenuation as a function of magnetic field. $T=4.2 \mathrm{~K}, v=9.3 \mathrm{GHz}$. Note that the vertical axis is inverted. it was not possible to define it with respect to the physical $a$ or $b$-axis.

The result is shown in figure 8 which was obtained for increasing $\theta$ from $0^{\circ}$ to $270^{\circ}$. Although an angular effect is clearly seen, it is not easy to determine its period. In particular, it is not found a $90^{\circ}$ period as would be expected if the two axes $a$ and $b$ actually were equivalent. An explanation of this behaviour, supported by the susceptibility experiments, is that there is actually a non equivalence between the two axes due to the non equivalent nucleation conditions of the domains according to the shape of the sample sides.

Furthermore, it is clear from the magnetization experiments that the transformation from the polydomain state to the monodomain is not reversible and that the monodomain state may be conserved when the magnetic field is reduced to zero.

So far as hypersonic experiments are concerned, large stresses are presumably induced in the sample due to the mounting. In addition, it was not possible to carry out the experiments for $\theta$ larger than $270^{\circ}$ because of ungluing probably caused by the presence of strong magnetic torques. Nevertheless, it is worth noticing that the sample itself was not damaged.

Previous measurements on the $\mathrm{RXO}_{4}$ compound $\mathrm{TbAsO}_{4}$ [23] have shown that for certain orientations the conversion to a monodomain state is not achieved even in strong magnetic fields. Similar effects may occur in our case and explain the behaviour of the $H_{\mathrm{p}}(\theta)$ curve above $180^{\circ}$.

\section{Conclusion.}

So far as the hypersonic experiments are concerned, the large noise observed in the high field region of the hysteresis cycle is attributed to the depinning of the domain walls.

The transformation from the polydomain to the monodomain state occurs in a field rather higher than in the magnetic measurements (Fig. 1). We think that this is due to the experimental set up which induces stresses in the sample.

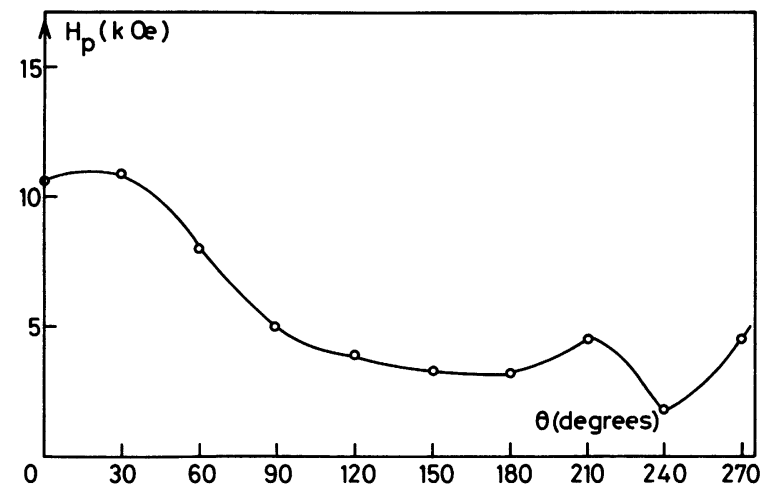

Fig. 8. $-H_{\mathrm{p}}$ (see Fig. 7) as a function of $\theta$ (see text). Note that the zero on $\theta$ axis is arbitrary. 
Our interpretation of the magnetic measurements is that they provide a tool for measuring the total relative ratio of the two-domain configurations in the sample. As a consequence, it is possible to analyse the evolution of this ratio when the sample is temperature cycled and to obtain information on the nucleation of the polydomain structure.

In particular it was possible to evidence geometry effects presumably due to differences in nucleation conditions.

On the other hand, it was possible to analyse the transformation of the polydomain sample into a monodomain. Both magnetization and hypersonic measurements support the phenomenological interpretation that we propose to account for the experiments. e.g. a process where the domains walls are progressively depinned and swept out of the sample to leave the monodomain structure.

\section{Acknowledgments.}

We thank M. Tur for experimental assistance. We are grateful to Profs J. Lajzerowicz, and M. Renard and to our colleagues for valuable discussions about this paper.

\section{References}

[1] Gehring, G. A., Gehring, K. A., Rep. Prog. Phys. 38 (1975) 1.

[2] Cooke, A. H., Martin, D. M., Wells, M. R., Solid. State Commun. 9 (1971) 519.

[3] Elliott, R. J., Harley, R. T., Hayes, W., Smith, S. R. P., Proc. R. Soc. A 328 (1972) 217.

[4] Cooke, A. H., Ellis, C. J., Gehring, K. A., Leask, M. J. M., Martin, D. M., WanKLYN, B. M., Wells, M. R., White, R. L., Solid State Commun. 8 (1970) 689.

[5] Leask, M. J. M., Maxwell, K. J., Tyte, R. N., Becker, P. J., Kasten, A., Wüchner, W., Solid State Commun. 13 (1973) 693.

[6] Pytte, E., Phys. Rev. B 9 (1974) 932.

[7] Suzuki, H., Ohtsuka, T., Yamadaya, T., Proceedings of the Low Temperature Physics Conference, LT 13, 2, 1974, p. 334.

[8] Picard, J., Hubsch, J., Le Gall, H., Guillot, M., J. Appl. Phys. 49 (1978) 1386.

[9] Kasten, A., Z. Physik 1338 (1980) 65.

[10] Hudson, R. P., Mangum, B. W., Phys. Lett. A 36 (1971) 157

[11] Smith, S. H., Garton, G., TANner, B. K., Midgley, D. J. Mat. Sciences 13 (1978) 620.
[12] Tanner, B. K., Smith, S. H., J. Cryst. Growth 28 (1975) 77.

[13] M'SiRDI, N., Thèse de 3e Cycle, Grenoble, 1983 and to be published.

[14] Gorobchenko, V. D., Lukashevich, I. I., StankeVICH, V. G., Mel'Nikov, E. V., Filippov, N. I., Sov. Phys. 14 (1973) 2140.

[15] Will, G., SCHÄFER, W., J. Phys. C. 4(1971) 811.

[16] SMith, S. H., private communication.

[17] PAGe, J. H., SMith, S. R. P., TAYloR, D. R., HARLEY, R. T., J. Phys. C. 12 (1979) L-875.

[18] Page, J. H., Taylor, D. R., Solid State Commun. 40 (1981) 907.

[19] Daudin, B., Thèse d'Etat, Grenoble, 1983, and to be published.

[20] Renard, M., Chouteau, G., Daudin, B., to be published.

[21] Kasten, A., BeCKer, P. J., Int. J. Magnetism 5 (1973) 157.

[22] Kasten, A., Müller, P. H., Schienle, M., Physica B 114 (1982) 77.

[23] Kasten, A., Berndts, P., Kahle, H. G., Physica B 80 (1975) 258. 